\title{
Notations and conventions in molecular spectroscopy: part 2. Symmetry notation
}

Article

Published Version

Schutte, C. J. H., Bertie, J. E., Bunker, P. R., Hougen, J. T., Mills, I., Watson, J. K. G. and Winnewisser, B. P. (1997)

Notations and conventions in molecular spectroscopy: part 2.

Symmetry notation. Pure and Applied Chemistry, 69 (8). pp.

1641-1649. ISSN 0033-4545 Available at

https://centaur.reading.ac.uk/7302/

It is advisable to refer to the publisher's version if you intend to cite from the work. See Guidance on citing.

Published version at: http://old.iupac.org/publications/pac/1997/

Publisher: IUPAC

All outputs in CentAUR are protected by Intellectual Property Rights law, including copyright law. Copyright and IPR is retained by the creators or other copyright holders. Terms and conditions for use of this material are defined in the End User Agreement.

\section{www.reading.ac.uk/centaur}

\section{CentAUR}

Central Archive at the University of Reading 
Reading's research outputs online 


\title{
INTERNATIONAL UNION OF PURE AND APPLIED CHEMISTRY
}

\author{
PHYSICAL CHEMISTRY DIVISION \\ COMMISSION ON MOLECULAR STRUCTURE AND SPECTROSCOPY* \\ SUBCOMMITTEE ON NOTATIONS AND CONVENTIONS FOR MOLECULAR SPECTROSCOPY

\section{NOTATIONS AND CONVENTIONS IN MOLECULAR SPECTROSCOPY: PART 2. SYMMETRY NOTATION}

(IUPAC Recommendations 1997)

\author{
Prepared for publication by \\ C. J. H. SCHUTTE 1 , J. E. BERTIE ${ }^{2}$, P. R. BUNKER ${ }^{3}$, J. T. HOUGEN ${ }^{4}$, I. M. MILLS ${ }^{5}$, \\ J. K. G. WATSON ${ }^{3}$ AND B. P. WINNEWISSER $^{6}$ \\ ${ }^{1}$ University of South Africa, PO Box 392, Pretoria 0001, South Africa \\ ${ }^{2}$ Department of Chemistry, University of Alberta, Edmonton, Canada T6G 2G2 \\ ${ }^{3}$ Steacie Institute for Molecular Sciences, National Research Council of Canada, Ottawa, K1A 0R6, Canada \\ ${ }^{4}$ Molecular Physics Division, National Institute for Standards and Technology, Gaithersburg, Maryland 20899, USA \\ ${ }^{5}$ Department of Chemistry, University of Reading, Whiteknights, Reading, RG6 2AD, UK \\ ${ }^{6}$ Justus Liebig Universität, Physikalisch-Chemisches Institut, Heinrich-Buff-Ring 58, D-6300 Giessen, Germany
}

* Membership of the Commission during the preparation of this report (1985-97) was as follows:

Chairman: J. R. Durig (USA; 1983-89); C. J. H. Schutte (RSA; 1989-93); J. E. Bertie (Canada; 1994-97); Secretary: H. A. Willis (UK; 1983-87); J. G. Grasselli (USA; 1987-89); J. F. Sullivan (USA; 1989-95); P. Klaeboe (Norway; 1996-97); Titular Members: A. M. Bradshaw (FRG; 1983-91); R. D. Brown (Australia; 1987-93); B. G. Derendjaev (Russia; 1983-91); R. K. Harris (UK; 1989-93); A. M. Heyns (RSA; 1994-97); E. Hirota (Japan; 1985-93); R. Janoschek (Austria; 1994-97); P. Klaeboe (Norway; 1994-97); J. F. J. Todd (UK; 1981-89); S. Tsuchiya (Japan; 1994-97); H. A. Willis (UK; 1983-87); B. P. Winnewisser (FRG; 1991-97); Associate Members: A. M. Bradshaw (FRG; 1991-95); S. M. Cabral de Menezes (Brazil; 1994-97); B. G. Derendjaev (Russia; 1991-95); P. Granger (France; 1989-93); R. K. Harris (UK; 1981-89); E. Hirota (Japan; 1994-97); J. Kowalewski (Sweden; 1996-97); S. Leach (France; 1987-91); G. J. Martin (France; 1983-89); J. P. Maier (Switzerland; 1989-93); L. N. Masalov (Russia; 1987-91); A. Oskam (Netherlands; 1991-97); W. B. Person (USA; 1983-89); C. J. H. Schutte (RSA; 1983-89); J. F. J. Todd (UK; 1989-91); S. Tsuchiya (Japan; 1987-93); C. Zhang (China; 1989-97); National Representatives: J. E. Collin (Belgium; 1985-97); S. M. Cabral de Menezes (Brazil; 1991-93); S. L. Spassov (Bulgaria; 1987-93); J. Lu (China; 1981-89); E. Hadjoudis (Greece; 1991-93); G. Varsányi (Hungary; 1985-93); M. Chowdhury (India; 1986-95); Z. Luz (Israel; 1983-93); S. Califano (Italy; 1989-97); S. Ng (Malaysia; 1983-93); A. Oskam (Netherlands; 1989-91); B. Jezowska-Trzebiatowska (Poland; 1983-89); J. J. C. Teixeira-Dias (Portugal; 1991-97); Y. S. Lee (Korea; 1989-97); T. A. Ford (RSA; 1987-91); C. Pascual (Spain; 1987-91); D. Escolar (Spain; 1991-97); J. Kowalewski (Sweden; 1994-95); M. Cebe (Turkey; 1987-91); S. Suzer (Turkey; 1991-93, 1995-97); S. Içli (Turkey; 1993-95); T. Ast (Yugoslavia; 1989-93); R. K. Harris (UK; 1993-97).

Republication or reproduction of this report or its storage and/or dissemination by electronic means is permitted without the need for formal IUPAC permission on condition that an acknowledgement, with full reference to the source along with use of the copyright symbol (C), the name IUPAC and the year of publication are prominently visible. Publication of a translation into another language is subject to the additional condition of prior approval from the relevant IUPAC National Adhering Organization. 


\title{
Notations and conventions in molecular spectroscopy: Part 2. Symmetry notation (IUPAC Recommendations 1997)
}

\begin{abstract}
The field of Molecular Spectroscopy was surveyed in order to determine a set of conventions and symbols which are in common use in the spectroscopic literature. This document, which is Part 2 in a series, establishes the notations and conventions used for the description of symmetry in rigid molecules, using the Schoenflies notation. It deals firstly with the symmetry operators of the molecular point groups (also drawing attention to the difference between symmetry operators and elements). The conventions and notations of the molecular point groups are then established, followed by those of the representations of these groups as used in molecular spectroscopy. Further parts will follow, dealing inter alia with permutation and permutation-inversion symmetry notation, vibration-rotation spectroscopy and electronic spectroscopy.
\end{abstract}

\section{PREAMBLE}

The sub-committee on Notations and Conventions in Molecular Spectroscopy was initiated by Commission I.5, the Commission on Molecular Structure and Spectroscopy, to establish and recommend a set of conventions and symbols from those that are in common use in the spectroscopic literature.

Part I of the series is titled General Spectroscopic Notation [1]. The present part deals with symmetry notation referring to point groups, and the recommendations follow those of Jenkins [2], Mulliken [3], IUPAP [4], and IUPAC [5] in the main. The notation of Herzberg [6] is recommended when it differs from that of Wilson, Decius and Cross [7]. It is pointed out that the International Union of Crystallography uses another approach [8].

\section{Part 2. SYMMETRY NOTATION}

2.1 Symmetry Operators for the Molecular Point Groups

2.2 The Molecular Point Groups

2.3 Representations for Molecular Point Groups and Spectroscopy

Symbols used in this paper

Greek Alphabet

$\begin{array}{llll}\Gamma & 2.3 .1 ; 2.3 .3 \mathrm{c} & \Sigma, \sigma & 2.3 .3 \mathrm{a} \\ \Gamma_{j}^{(\alpha)} & 2.3 .2 & \sigma & 2.1 .2 \mathrm{a} \\ \Gamma, \gamma & 2.3 .3 \mathrm{c} & \sigma_{\mathrm{h}} & 2.1 .2 \mathrm{~b} \\ \Delta, \delta & 2.3 .3 \mathrm{c} & \sigma_{\mathrm{v}}, \sigma_{\mathrm{v}}{ }^{\prime} & 2.1 .2 \mathrm{c} \\ \Pi, \pi & 2.3 .3 \mathrm{c} & \sigma_{\mathrm{d}}, \sigma_{\mathrm{d}}{ }^{\prime} & 2.1 .2 \mathrm{~d} ; 2.1 .7 \\ \Phi, \phi & 2.3 .3 \mathrm{c} & \sigma_{x y}, \sigma(x, y) & 2.1 .6 \\ \Omega & 2.3 .11 & & \end{array}$




$\begin{array}{llll}\text { matrices } & 2.2 .17 & 1,2 & 2.3 .6 ; 2.3 .9 ; 2.3 .12 \mathrm{a} \\ \text { ', } & 2.1 .7 ; 2.3 .7 & +- & 2.3 .13 \\ \text { upper/lower case } & 2.3 .4 & & \end{array}$

\begin{tabular}{|c|c|c|c|}
\hline A & 2.3.3a; 2.3 .12 & $\mathrm{G}$ & $2.3 .3 \mathrm{e} ; 2.3 .12$ \\
\hline $\mathrm{a}$ & $2.3 .3 \mathrm{a}$ & $\mathrm{g}$ & $2.3 .3 \mathrm{e} ; 2.3 .5 \mathrm{a}$ \\
\hline B & $2.3 .3 \mathrm{~b} ; 2.3 .12$ & $\mathrm{H}, \mathrm{h}$ & $2.3 .3 f$ \\
\hline $\mathrm{b}$ & $2.3 .3 \mathrm{~b}$ & $I, \mathrm{I}$ & $2.1 .1 ; 2.3 .12$ \\
\hline$C_{n}, C_{n}{ }^{1}$ & $2.1 .3 ; 2.2 .1$ & $I_{\mathrm{h}}$ & 2.2 .13 \\
\hline$C_{n}^{k}, C_{n}^{k}(x)$ & $2.1 .3 ; 2.1 .6$ & $i$ & 2.1 .5 \\
\hline$C_{2}, C_{2}^{\prime}, C_{2}^{\prime \prime}$ & 2.1 .7 & $\ell$ & 2.3 .8 \\
\hline$C_{\mathbf{i}}$ & 2.2 .2 & $m$ & 2.3 .10 \\
\hline$C_{n v}$ & 2.2 .6 & $O$ & 2.2 .11 \\
\hline$C_{n \mathrm{~h}}, C_{1 \mathrm{~h}}$ & 2.2 .7 & $O_{\mathrm{h}}$ & 2.2 .12 \\
\hline$C_{\mathrm{s}}$ & 2.2 .7 & $S_{n}, S_{n}{ }^{1}$ & 2.1 .4 \\
\hline$C_{\infty}(\phi)$ & 2.1 .3 & $S_{\infty}(\phi)$ & 2.1 .4 \\
\hline$C_{\infty \mathrm{vv}}$ & 2.2 .14 & $S_{n}^{k}, S_{2}{ }^{1}$ & $2.1 .4 ; 2.1 .5$ \\
\hline$D_{n}, D_{2}$ & 2.2 .3 & $S_{2 n}, S_{2}$ & 2.2 .2 \\
\hline$D_{n \mathrm{~h}}, D_{2 \mathrm{~h}}$ & 2.2 .4 & $T, \mathrm{~T}$ & $2.2 .8 ; 2.3 .3 \mathrm{~d}$ \\
\hline$D_{n \mathrm{~d}}, D_{2 \mathrm{~d}}$ & 2.2 .5 & $T_{\mathrm{d}}$ & 2.2 .10 \\
\hline$D_{\infty \mathrm{h}}$ & 2.2 .15 & $T_{\mathrm{h}}$ & 2.2 .9 \\
\hline$E, \mathrm{E}$ & $2.1 .1 ; 2.3 .3 c ; 2.3 .12$ & $\mathrm{t}$ & $2.3 .3 \mathrm{~d}$ \\
\hline$e, \mathrm{e}$ & $2.1 .1 ; 2.3 .3 \mathrm{c}$ & u & $2.3 .5 b$ \\
\hline $\mathrm{F}$ & $2.3 .3 \mathrm{~d} ; 2.3 .12$ & $x, y, z$ & 2.1 .6 \\
\hline$f$ & $2.3 .3 \mathrm{~d}$ & & \\
\hline
\end{tabular}




\section{Typographical Conventions:}

It is desirable to distinguish by means of typographic conventions between the symmetry elements of a body (such as, for example, the invariant line called a rotation axis) and the symmetry operators (sometimes called symmetry operations) which bring a body into coincidence with itself and which form a point group. Unfortunately there seems to be no established set of typographic conventions for doing so. The conventions used in this paper are stated below. It is recommended that authors who do not follow these conventions clearly define their own conventions.

Symbols for symmetry operators are printed italic, with sub- and super-scripts that are upright except for the variables $k$ and $n$ which are replaced by numbers for a specific operator [5]. Following Donnay (Acta Cryst. A28, (1972) 110), the power of an operator, $k$, is specified even when it is one, e.g., $C_{n}{ }^{1}$ is used instead of $C_{n}$; and $S_{n}{ }^{1}$ is used instead of $S_{n}$.

Symbols for symmetry elements are seldom used in this document. Those that are included are printed upright.

Symbols for symmetry groups are printed italic with upright subscripts, following common usage.

Symbols for irreducible representations of point groups, which are usually called symmetry species in spectroscopy, are printed upright. Subscripts are upright except when they are the variables $j$ or $\alpha$ which are replaced by numbers in specific cases. $\Gamma$ is printed italic when it is a variable to be replaced by other symbols in specific cases.

\subsection{SYMMETRY OPERATORS OF THE MOLECULAR POINT GROUPS}

The symbols for symmetry operators are defined below. Only the Schoenflies notation is followed here. The operator symbols approved by the International Union of Crystallography [8] for use in crystal chemistry, which include translational symmetry operators, differ from those given here.

The correspondences are $(E \equiv I),\left(C_{n}{ }^{1} \equiv n\right)(i \equiv \bar{l}),(\sigma \equiv m),\left(S_{n}{ }^{1} \equiv \bar{n}\right.$ or $\left.\bar{X}\right)$;. A table showing the alternative notations of the point groups used by crystallographers is found in Ref. 8, p. 801, as well as in Schutte [9], p. 393.

\subsubsection{Identity operator}

$$
E ; e ; I
$$

\subsubsection{Reflection operator for reflection across a plane}

(a) across a general plane

$\sigma$

(b) across a horizontal plane

(c) across a vertical plane

(d) in a plane bisecting two $\mathrm{C}_{2}$-axes which are perpendicular to the principal $\mathrm{C}_{n}$ axis

$$
\begin{aligned}
& \sigma_{\mathrm{h}} \\
& \sigma_{\mathrm{v}} \\
& \sigma_{\mathrm{d}}
\end{aligned}
$$

$C_{n}^{k} ; C_{n}^{1}=C_{n}$

$C_{\infty}(\phi)$ 
2.1.4 $n$-fold rotation-reflection (alternating; improper) operator for $k$ successive rotation-reflections about an $n$-fold rotationreflection axis for a rotation through an angle of $2 \pi / n$ followed by a reflection in a plane perpendicular to the axis, where $n=2,3,4, \ldots ; k=1,2, \ldots(n-1)$

or: $n=\infty \quad$; rotation through an arbitrary angle $\phi$

$S_{n}^{k} ; S_{n}^{1}=S_{n}$

$S_{\infty}(\phi)$

$i$, note $S_{2}{ }^{1} \equiv i$

2.1.5 Inversion operator through the centre of symmetry

2.1.6 Specification of orientation: If it is needed to specify the orientation of the symmetry element with respect to a moleculefixed set of cartesian axes $(x, y, z)$ which is involved in the symmetry operation, an appropriate subscript or symbols in brackets may be added to the symbol of the symmetry operation, e.g., $\sigma_{x y}, \sigma(x y), C_{n}^{k}(x)$

2.1.7 Distinguishing between same kind of operators: symmetry operators of the same kind, but belonging to different group classes must be distinguished by consecutive right accent superscripts,

e.g., $C_{2}, C_{2}{ }^{\prime}, C_{2}{ }^{\prime \prime}$, and $\sigma_{\mathrm{d}}$ and $\sigma_{\mathrm{d}}{ }^{\prime}$ in $D_{4 \mathrm{~h}}$

\subsection{THE MOLECULAR POINT GROUPS}

The essential operators in each point group are listed. Other operators are present in the groups marked $* *$, required by the presence of those listed. References 6 and 7 give a complete listing of the operators in each group.

2.2.1 Finite rotation groups having only $C_{n}{ }^{k}$ operators $C_{n}$

2.2.2 Rotation-reflection groups having only $S_{2 n}{ }^{k}$ operators

$$
S_{2 n} ; S_{2} \equiv C_{\mathrm{i}}
$$

2.2.3 Group of operators of the group $C_{n}$ plus $n$-dihedral $C_{2}$ operators

$D_{n} ; D_{2}$

2.2.4 Group of operators of the group $D_{n}$ plus the $\sigma_{\mathrm{h}}$ operator**

$D_{n \mathrm{~h}} ; D_{2 \mathrm{~h}}$

2.2.5 Group of operators of the group $D_{n}$ plus $n \sigma_{\mathrm{d}}$ operators**

$D_{n \mathrm{~d}} ; D_{2 \mathrm{~d}}$

2.2.6 Group of operators of the group $C_{n}$ plus $n \sigma_{\mathrm{v}}$ operators

$C_{n \mathrm{v}}$

2.2.7 Group of operators of the group of $C_{n}$ plus the $\sigma_{\mathrm{h}}$ operator**

$C_{n h} ; C_{1 \mathrm{~h}} \equiv C_{\mathrm{s}}$

2.2.8 Group of rotation operators of a tetrahedron

$T$

2.2.9 Group of operators of $T$ plus the inversion operator $i \quad T_{\mathrm{h}}$

2.2.10 Full group of operators of a tetrahedron $\quad T_{\mathrm{d}}$

2.2.11 Group of operators for the rotations of an octahedron (isomorphous with $T_{\mathrm{d}}$ )

2.2.12 Full group of operators of an octahedron (Group of operators of $O$ plus the inversion operator $i$ )

$O_{\mathrm{h}}$ 
2.2.13 Full group of operators for an icosahedron $\quad I_{h}$

2.2.14 Group of operators for an asymmetric linear molecule $\quad C_{\infty \mathrm{V}}$

2.2.15 Group of operators for a symmetric linear molecule (the group of operators of $C_{\infty \mathrm{v}}$ plus the inversion operator $\left.i\right)^{* *} \quad D_{\infty \mathrm{ch}}$

2.2.16 Alternative definitions: Some of the point groups may be defined in an alternative fashion as direct product groups with the $i$-operator, e.g., for $n$ odd $C_{n} \times i=S_{2 n}$. The $C_{n \mathrm{~h}}$ groups are also generated by $C_{n} \times \sigma_{\mathrm{h}}=C_{n \mathrm{~h}}$ or for $n$ even by $C_{n} \times i=C_{n \mathrm{~h}}$

2.2.17 Matrices representing symmetry operators: the matrices representing the symmetry operators of a group $G$ are indicated by the corresponding heavy-print symbols. Definitions should include whether the operators are active (operating on the position vectors) or passive (operating on the molecule-fixed coordinate system), whether the rotations are clockwise or anticlockwise with respect to the positive direction of the rotation axis, and the handedness of the axial system.

\subsection{REPRESENTATIONS FOR MOLECULAR POINT GROUPS AND SPECTROSCOPY}

\subsubsection{Reducible representation of group $G$}

2.3.2 Irreducible representations of group $G$, where $j=1,2,3, \ldots$ numbers the representations sequentially and $(\alpha)=1,2,3$ indicates the dimension of each representation $\Gamma_{j}^{(\alpha)}$ alternatively, the symbols of 2.3.3 may replace $\Gamma_{j}^{(\alpha)}$ $A_{1 \mathrm{~g}}, F_{2 u}$, etc.

2.3.3 The dimensions of the representations are indicated by the following symbols (which may be sub- and superscripted according to $2.3 .5-2.3 .12$ ), the choice between upper case and lower case letters being regulated by 2.3 .4 :

(a) one-dimensional, symmetric $(+1)$ with respect to the primary operator $C_{n}{ }^{1}$ or $S_{n}{ }^{1}$ of the point group

polyatomic linear

(b) one-dimensional, antisymmetric (-1) with respect to the primary operator $C_{n}{ }^{1}$ or $S_{n}{ }^{1}$ of the point group
A, a
$\Sigma, \sigma$

(c) two-dimensional and pseudo two-dimensional

$\mathrm{B}, \mathrm{b}$

E, e

$\Pi, \pi$

$\Delta, \delta$

$\Phi, \phi$

$\Gamma, \gamma$

Note: The complex conjugate one-dimensional representations $\Gamma_{i}$ and $\Gamma_{j}$ are bracketed together in spectroscopy as a pseudo E-representation or corepresentation, because the Hamiltonian is invariant to the operator which expresses time-reversal, that is, the wavefunction and its complex conjugate yield the same energy. This occurs, for instance, in the groups $C_{n}, S_{2 n}$, and $C_{n h}$ 
(d) three-dimensional (the symbol $\mathrm{T}$ is customarily used in the context of inorganic complexes, to avoid confusion with $\mathrm{F}$ for atomic states in the full rotation group)

$\mathrm{F}, \mathrm{f} ; \mathrm{T}, \mathrm{t}$

(e) four-dimensional

$\mathrm{G}, \mathrm{g}$

(f) five-dimensional

$\mathrm{H}, \mathrm{h}$

2.3.4 Symbols for symmetry species in 2.3.3 are used upper case or lower case as follows:

(a) Individual normal vibration wavefunction

lower case

(b) Overall vibrational state wavefunction

(c) Individual one-electron (orbital) wavefunction

upper case

(d) Overall electronic state wavefunction

lower case

upper case

(e) Overall vibronic state wavefunction

upper case

2.3.5 Right subscripts on the representations of 2.3.3 with respect to the inversion operator $i$ :

(a) representations which are symmetric $(+1)$ with respect to the inversion operator $i$

(b) representations which are antisymmetric $(-1)$ with respect to the inversion operator $i$

2.3.6 Right subscripts on the representations A and/or B of 2.3.3 for the groups $D_{n}, D_{n \mathrm{~h}}$ and $D_{n \mathrm{~d}}$ or for $C_{n \mathrm{v}}$

(a) representations which are symmetric with respect to the $C_{2}{ }^{\prime}$ operator for a rotation around a $C_{2}{ }^{\prime}$ axis $\perp$ to the generating axis $C_{n}$ of the groups $D_{n}, D_{n \mathrm{~h}}$, and $D_{n \mathrm{~d}}$

(b) representations which are symmetric with respect to the $\sigma_{\mathrm{v}}$ operator in the group $C_{n \mathrm{v}}$

(c) representations which are antisymmetric with respect to the $C_{2}{ }^{\prime}$ operator in the groups $D_{n}, D_{n \mathrm{~h}}$, and $D_{n \mathrm{~d}}$

(d) representations which are antisymmetric with respect to the $\sigma_{\mathrm{v}}$ operator in the groups $C_{n \mathrm{v}}$

Note: To resolve the ambiguity in assigning the subscript labels of the B species of the point groups $C_{2 \mathrm{v}}$ and $D_{2 \mathrm{~h}}$ for planar molecules, the $x$ axis must be chosen perpendicular to the plane of the molecule. For $D_{2 \mathrm{~h}}$ the $z$ axis is chosen so that it passes through the largest number of atoms or, if this is not unique, then so that it cuts the largest number of bonds [3]. The Mulliken [3] numbering of the $B_{1}, B_{2}$ and $\mathrm{B}_{3}$ species of $D_{2 \mathrm{~h}}$ is followed.

2.3.7 Right superscripts on the representations of 2.3.3 with respect to the $\sigma_{\mathrm{h}}$ operator

(a) representations which are symmetric with respect to the $\sigma_{\mathrm{h}}$-operator

(b) representations which are antisymmetric with respect to the $\sigma_{\mathrm{h}}$-operator 
2.3.8 Right subscript $\ell$ on the E-representation where $\ell=1,2,3, \ldots$ $\left(\frac{1}{2} n-1\right)$ for $n$ even and $\frac{1}{2}(n-1)$ for $n$ odd, where $n$ is the order of the generating operator of the point group

2.3.9 Right subscripts 1 and 2 on F-representations of the groups $T_{\mathrm{d}}, O, O_{\mathrm{h}}$

Note: Since the use of 1 or 2 is arbitrary, the notation of Herzberg [6] and Wilson, Decius and Cross [7] is recommended

2.3.10 A left superscript $m$ to indicate electron spin multiplicity $m=2 S+1$ may be added to the species symbols of 2.3.4(d) and (e); for example, ${ }^{3} \mathrm{~A}_{2},{ }^{3} \Sigma_{\mathrm{g}}^{-}$

2.3.11 A right subscript $\Omega$ or $P$ of 1.2.2.16 [1] may be added to the species symbols of linear molecules, for example, ${ }^{2} \Pi_{(3 / 2) \mathrm{u}},{ }^{2} \Delta_{5 / 2} \quad \Omega$

2.3.12 Level designations according to the value of $\Omega$ for very strong spin-orbit coupling (Hund's case c):

(a) diatomic and linear molecules odd electron states* even electron states* $\frac{1}{2}, \frac{3}{2}, \frac{5}{2}, \ldots$ $0^{+}, 0^{-}, 1,2, \ldots$ (b) non-linear molecules odd electron states $* *+$ even electron states**+ $E_{1 / 2}, E_{3 / 2}, \ldots G_{5 / 2}, I_{5 / 2}$
$A_{1}, A_{2}, B_{1}, B_{2}, E_{1}, E_{2}, F_{1} \ldots$

2.3.13 Right superscripts on the $\Sigma$ representations of the groups $C_{\infty \mathrm{V}}$ and $D_{\infty \mathrm{h}}$

(a) representations which are symmetric with respect to the reflection operator $\sigma_{\mathrm{v}}$

(b) representations which are antisymmetric with respect to the reflection operator $\sigma_{\mathrm{v}}$

*For $D_{\infty \mathrm{h}}$ add the $\mathrm{g}$, u subscripts.

**For $D_{6 \mathrm{~h}}$ and $O_{\mathrm{h}}$ add the $\mathrm{g}$, u subscripts, and for $D_{3 \mathrm{~h}}$ and $C_{3 \mathrm{~h}}$ add the ' and " superscripts, omitting the 1 and 2 subscripts.

${ }^{+}$Multiply the orbital symmetry species by the spin symmetry species to obtain the appropriate level designation, keeping in mind the ' and " signs, as well as g and $\mathrm{u}$.

\section{REFERENCES}

1 C. J. H. Schutte, J. E. Bertie, P. R. Bunker, J. T. Hougen, I. M. Mills, J. K. G. Watson and B. P. Winnewisser,(Part 1), Pure Appl. Chem., this issue (1997).

2 F. A. Jenkins, Phys. Rev. 43, (1953) 425.

3 Report on Notation for the Spectra of Polyatomic Molecules, prepared for the Joint Commission for Spectroscopy by R. S. Mulliken, J. Chem. Phys., 23, (1955) 1997.

4 IUPAP, Symbols, Units, Nomenclature and Fundamental Constants in Physics, 1987 Revision, E. R. Cohen and P. Giacomo, Document IUPAP-25 (SUNAMCO 87-1), Physica, $146 A$ (1987) 1. 
5 I. Mills, T. Cvitas, K. Homann, N. Kallay and K. Kuchitsu, Quantities, Units and Symbols in Physical Chemistry, International Union of Pure and Applied Chemistry (Blackwell Scientific Publications, Oxford, 1993) Second Edition.

6 G. Herzberg, Molecular Spectra and Molecular Structure: II Infrared and Raman Spectra of Polyatomic Molecules, D. Van Nostrand Company, Inc., Princeton 1945; III Electronic Spectra and Electronic Structure of Polyatomic Molecules, D. Van Nostrand Company, Inc., Princeton 1966. Reprinted by Krieger Publishing Co., Malabar, Fl. (1991).

7 E. B. Wilson, Jr., J. C. Decius and P. C. Cross, Molecular Vibrations, McGraw-Hill Publishing Co., New York, 1955.

8 T. Hahn, International Tables for Crystallography, Volume A, Fourth Revised Edition, Kluwer Academic Publishers, Dordrecht, 1995.

9 C. J. H. Schutte, The Theory of Molecular Spectroscopy, Volume 1. The Quantum Mechanics and Group Theory of Vibrating and Rotating Molecules. North Holland, Amsterdam/American Elsevier, New York, 1976. 